\title{
DIVERSIDADE DE BETHYLIDAE (HYMENOPTERA) DO PARQUE NACIONAL DA SERRA DO DIVISOR, ACRE, BRASIL E SEU USO NO PLANO DE MANEJO E CONSERVAÇÃO DA ÁREA
}

\author{
Celso Oliveira AZEVEDO ${ }^{1}$, José Luiz HELMER ${ }^{1}$, Elder MORATO ${ }^{2}$
}

\begin{abstract}
Resumo - Foram utilizadas prioridades de conservação de morfo-espécies e gêneros, importância relativa de sítios e prioridade média para estabelecer um modelo de conservação para o Parque Nacional Serra do Divisor no Estado do Acre, Brasil, baseado na fauna de Bethylidae. Foram instaladas armadilhas Malaise em 12 sítios amostrais nas regiões norte em novembro de $1996 \mathrm{e}$ sul do março de 1997, sendo reconhecidas 102 morfo-espécies de 8 gêneros de Bethylidae, com maior diversidade na região norte do parque. As morfo-espécies exclusivas foram os táxons com maior prioridade de conservação. Os sítios S6, N9, N6 e N5 apresentaram os índices mais altos de importância relativa para conservação por terem sido mais diversos.
\end{abstract}

Palavras-chave: Bethylidae, Brasil, biologia da conservação, Hymenoptera, Parque Nacional da Serra do Divisor.

Diversity of Bethylid Wasps (Insecta, Hymenoptera) and their Use on the Management and Conservation Plan for the Serra do Divisor National Park, Acre, Brazil

Abstract - Malaise traps were set at 12 sampling sites in November, 1996 in the northern and in March, 1997 in the southern regions of Serra do Divisor National Park. One hundred and two morphospecies were recognized, belonging to 8 genera of Bethylidae. Diversity of these wasps was higher at the northern region of the park. Conservation priority of genera and morphospecies, relative importance of areas and average (conservation) priority for species was employed to propose a conservation model for the park, based on Bethylidae fauna. The conservation of the exclusive species was considered priority. Sampling sites S6, N9, N6 and N5 presented the highest indices of relative importance due to this high species diversity.

Key-words: Bethylidae, Brazil, Conservation Biology, Hymenoptera, Serra do Divisor National Park.

\section{INTRODUÇÃO}

O Parque Nacional da Serra do Divisor (PNSD) está localizado na região noroeste do Acre, no alto do vale do rio Juruá, na divisa com o Peru, incluindo cinco municípios. A caça comercial, extração de madeira, desmatamentos, caminho para tráfico de drogas e extração de fósseis são as principais ameaças que o Parque vem sofrendo.
Para tentar conter tais problemas a S.O.S. Amazônia, The Nature Conservancy e IBAMA/UFAC elaboraram o "Projeto para a Conservação do Parque Nacional da Serra do Divisor", que teve como objetivo elaborar um plano de manejo para o parque com o intuito de estabelecer estratégias de preservação de sua biodiversidade. Para tanto, contaram com a colaboração de pesquisadores de diversas instituições, principalmente,

'Universidade Federal do Espírito Santo, Departamento de Biologia, Av. Marechal Campos 1468, Maruípe, 29.040-090 Vitória, ES, Brasil (cazevedo@npd.ufes.br)

${ }^{2}$ Universidade Federal do Acre, Departamento de Ciências da Natureza, 69.915-900 Rio Branco, Acre, Brasil. Endereço atual: Universidade Federal de Minas Gerais, PPG-Ecologia, Conservação e Manejo da Vida Silvestre30.161-970 Belo Horizonte, Minas Gerais (morato@mono.icb.ufmg.br). 
para a identificação dos táxons.

Tradicionalmente, as propostas de manejo e conservação de áreas silvestres são fundamentadas em estudos realizados com vertebrados ou plantas vasculares (New, 1999; Oliver \& Beattie, 1993, 1996). A ausência de estudos com esta abordagem usandose animais invertebrados e plantas sem flores tem sido justificada pela dificuldade de identificação dos táxons, custo e tempo (Oliver \& Beattie, 1993, 1996), mas em função da alta diversidade de espécies de insetos, estes podem ser usados como ferramenta ecológica para monitoramento de qualidade ambiental (New, 1999).

Os Bethylidae

são ectoparasitóides de larvas de lepidópteros e, principalmente, de coleópteros que vivem em ambientes crípticos (Evans, 1964; Azevedo, 1999). Himenópteros parasitóides participam em mais da metade das cadeias alimentares em ambientes terrestres e regulam a densidade de populações de seus insetos hospedeiros, promovendo o equilíbrio das comunidades envolvidas (LaSalle \& Gauld, 1995). Além disso, parasitóides de hospedeiros protegidos ou ocultos são mais diversos em regiões tropicais (Rathcke \& Price, 1976). Deste modo, os Bethylidae parecem possuir caraterísticas capazes de indicar áreas com padrões diferentes de biodiversidade.

Este trabalho teve como objetivo contribuir com a proposta de manejo e conservação do Parque Nacional da Serra do Divisor, usando-se vespas da família Bethylidae (Insecta, Hymenoptera) como base para reconhecer áreas do Parque com maior riqueza de espécies, que juntos com diversos outros estudos ali realizados, poderão estabelecer ações de manejo e conservação para o Parque.

\section{MATERIAL E MÉTODOS}

O levantamento de campo foi realizado no Parque Nacional da Serra do Divisor (PNSD). Situado a noroeste do estado do Acre, na fronteira com o Peru, Amazônia Ocidental, com as coordenadas de longitudes entre $74^{\circ} 00^{\prime}$ e $72^{\circ} 45^{\prime} \mathrm{W}$ e latitudes entre $7^{\circ} 15^{\prime}$ e $9^{\circ} 04^{\prime} \mathrm{S}$, o PNSD possui uma área de 843.000 ha. Dele fazem parte os municípios de Cruzeiro do Sul, Mâncio Lima, Rodrigues Alves, Porto Walter e Marechal Thaumaturgo.

O clima na região do PNSD é quente e úmido, com temperatura média anual igual a $24,2^{\circ} \mathrm{C}$, máxima média anual igual a $37^{\circ} \mathrm{C}$ e mínima média anual igual a $5^{\circ} \mathrm{C}$. Na região sul contudo, as temperaturas são menos elevadas; a umidade relativa do ar varia entre 80 e $90 \%$ ao longo do ano; a pluviosidade varia entre $1.750 \mathrm{e}$ $2.000 \mathrm{~mm}$ anuais e não existe uma estação seca definida. Os meses menos chuvosos são junho, julho e agosto; os mais chuvosos estão entre outubro e abril (Brasil, 1977).

O relevo, da maior parte do PNSD, é colinoso com altitudes variando entre 200 e $300 \mathrm{~m}$; na extremidade oeste ocorre uma zona de montanhas, o complexo fisiográfico da Serra do Divisor, o qual alcança altitude máxima de $580 \mathrm{~m}$. 
Os rios mais importantes são o Moa e Azul (região norte), e o Juruá Mirim (e seu afluente Rio Branco), Ouro Preto, das Minas, todos afluentes do Rio Juruá na sua margem esquerda (região sul). O rio Juruá é o mais importante e tem o Moa como seu principal afluente na sua margem esquerda.

A maior parte da região possui vegetação do tipo floresta ombrófila aberta de palmeiras, de bambus e de cipós; aproximadamente um terço da área possui floresta ombrófila densa: floresta sub-montana e floresta densa dos baixos platôs (Brasil, 1977).

A amostragem foi realizada em 12 sítios de coleta, situados no in- terior de oito diferentes tipologias de vegetação (Tab. 1).

Devido a grande extensão do PNSD a amostragem foi dividida em duas etapas. A primeira ocorreu em novembro de 1996 na região norte do parque, com acesso principalmente pelos rios Moa e Azul; a segunda ocorreu em março de 1997 na região sul, com acesso através dos rios Juruá, Juruá Mirim, Branco, Ouro Preto e das Minas.

$O$ esforço de coleta foi padronizado em todos os sítios amostrados, através de armadilha Malaise, instalada em cada tipologia de vegetação e operada por 24 horas. Ao todo, foram realizadas cerca de 288 horas de amostragem.

Tabela 1. Características dos sítios amostrais do Parque Nacional da Serra do Divisor, $\mathrm{N}=$ norte, $\mathrm{S}=$ sul, segundo Brasil (1977).

\begin{tabular}{|c|c|c|}
\hline Sitio & Tipologia & Coordenadas \\
\hline Ni & Floresta densa sub-montana & $7^{\circ} 26^{\prime} 36^{\prime \prime} \mathrm{S}, 73^{\circ} 40^{\prime} 28^{\prime \prime} \mathrm{W}$ \\
\hline N3 & Floresta densa sub-montana & $7^{\circ} 28^{\prime} 38^{\prime \prime} \mathrm{S}, 73^{\circ} 41^{\prime} 54^{\prime \prime} \mathrm{W}$ \\
\hline N5 & Floresta densa nos platôs & $7^{\circ} 27^{\prime} 07^{\prime \prime} \mathrm{S}, 73^{\circ} 47^{\prime} 06^{\prime \prime} \mathrm{W}$ \\
\hline N6 & Floresta aberta de palmeiras em depósitos coluviais & $7^{\circ} 26^{\prime} 27^{\prime \prime} \mathrm{S}, 73^{\circ} 39^{\prime} 28^{\prime \prime} \mathrm{W}$ \\
\hline N8 & Floresta aberta de palmeiras nos platôs & $7^{\circ} 33^{\prime} 24^{\prime \prime} \mathrm{S}, 73^{\circ} 16^{\prime} 36^{\prime \prime} \mathrm{W}$ \\
\hline N9 & Floresta de várzea & $7^{\circ} 27^{\prime} 02^{\prime \prime} \mathrm{S}, 73^{\circ} 36^{\prime} 30^{\prime \prime} \mathrm{W}$ \\
\hline S1 & $\begin{array}{l}\text { Floresta aberta de palmeiras com bambu dominante sobre a } \\
\text { formação Solimōes do Pleistoceno }\end{array}$ & $8^{\circ} 16^{\prime} 51^{\prime \prime}$ S, $73^{\circ} 15^{\prime} 13^{\prime \prime} \mathrm{W}$ \\
\hline $\mathrm{S} 4$ & $\begin{array}{l}\text { Floresta aberta de palmeiras sobre a formação Solimôes do } \\
\text { Pleistoceno }\end{array}$ & $8^{\circ} 24^{\prime} 19^{\prime \prime} \mathrm{S}, 72^{\circ} 51^{\prime} 28^{\prime \prime} \mathrm{W}$ \\
\hline S6 & Floresta aberta de palmeiras nos terraços altos & $8^{\circ} 33^{\prime} 30^{\prime \prime} \mathrm{S}, 72^{\circ} 53^{\prime} 30^{\prime \prime} \mathrm{W}$ \\
\hline S8 & $\begin{array}{l}\text { Vegetação de taboca em floresta aberta de palmeiras nos terraços } \\
\text { altos }\end{array}$ & $8^{\circ} 33^{\prime} 50^{\prime \prime} \mathrm{S}, 72^{\circ} 51^{\prime} 45^{\prime \prime} \mathrm{W}$ \\
\hline S10 & $\begin{array}{l}\text { Floresta aberta de palmeiras com bambu dominante sobre a } \\
\text { formação Solimôes }\end{array}$ & $8^{\circ} 52^{\prime} 31^{\prime \prime}$ S, $72^{\circ} 46^{\prime} 55^{\prime \prime} \mathrm{W}$ \\
\hline S11 & Floresta de várzea & $8^{\circ} 51^{\prime} 37^{\circ} \mathrm{S}, 72^{\circ} 52^{\prime} 20^{\prime \prime} \mathrm{W}$ \\
\hline
\end{tabular}


Todo o material coletado na primeira e segunda etapas de amostragem foi inicialmente trabalhado no Laboratório de Entomologia do Departamento de Ciências Agrárias (DCA) da Universidade Federal do Acre (UFAC).

Os Bethylidae obtidos foram triados em gêneros e morfo-espécies, seguindo metodologia de Oliver \& Beattie (1996). Dados de identificação deste material com alguns gêneros, como Dissomphalus Ashmead e Apenesia Westwood, indicam que uma porcentagem significativa do material estudado corresponde a espécies não descritas.

Através das equações abaixo (Minns, 1987) foram estabelecidas prioridade de conservação de cada táxon $\left(Q_{j}\right)$, importância relativa de sítios $\left(I_{i}\right)$ e prioridade média entre táxons e sítios $\left(Q_{m i}\right)$ no interior do Parque:

$$
\begin{aligned}
& Q_{j}=1-\sum_{i=1}^{n} \mathrm{~S}_{i j} \mathrm{~W}_{i} / \sum_{i=1}^{n} \mathrm{~W}_{i} \\
& I_{i}=\sum_{j=I}^{m} \mathrm{~S}_{i j} \mathrm{Q}_{j} / \sum_{j=1}^{m} \mathrm{Q}_{j} \\
& Q_{m i}=\sum_{j=I}^{m} \mathrm{~S}_{i j} \mathrm{Q}_{\mathrm{j}} / \sum_{j=I}^{m} \mathrm{~S}_{i j}
\end{aligned}
$$

onde $n=$ número de sítios, $m$ = número de táxons, $S_{i j}=$ presença (1) ou ausência (0) do táxon $j$ no sítio $i$ e $W_{i}=$ peso do sítio $i$, igual a um (1) para cada sítio, pois considerou-se que as importâncias deles eram iguais. Pesos diferenciados poderiam ser usados se o pesquisador considerar que as importâncias sob o ponto de vista de conservação devem ser diferentes. Todos os coeficientes, $Q_{j}, I_{i}$ e $Q_{m i}$ possuem valores que variam entre 0 e 1.

Foi apresentado um índice médio, $\left(I_{i}+Q_{m i}\right) / 2$, que representa uma ponderação entre importância relativa de sítios e prioridade de táxons e sítios. Esta média atenua possíveis efeitos de dados discrepantes de um único índice e mostra uma tendência mais global do ambiente.

Em nosso trabalho tanto as coletas como o reconhecimento das morfo-espécies foram feitas por especialistas em Hymenoptera, uma vez que o uso de pessoas não especialistas em taxonomia para amostragem em campo e triagem das morfo-espécies pode trazer risco, como alertado por Goldstein (1997).

\section{RESULTADOS E DISCUSSÃO}

Foram obtidos 353 espécimes de Bethylidae. Considerando-se que o esforço amostral total foi de 12 dias de exposição de armadilha Malaise, obteve-se uma média alta de 29,4 espécimes/dia/armadilha, quando comparado com Noyes (1989) que obteve 2,66 espécimes/dia/armadilha em uma floresta tropical úmida na Indonésia.

Foram reconhecidas distribuídas em 8 gêneros e 102 morfo-espécies de Bethylidae no PNSD, Anisepyris Kieffer, Apenesia Westwood, Dissomphalus Ashmead, Epyris Westwood, Goniozus Förster, 
Holepyris Kieffer, Pseudisobrachium Kieffer e Rhabdepyris Kieffer, dos quais os sete primeiros são aqui citados pela primeira vez para o Estado do Acre. Os gêneros Dissomphalus e Pseudisobrachium foram os mais abundantes e diversificados, com 30 e 29 morfoespécies, respectivamente, tendo ocorrido em quase todos os sítios amostrais, exceto no sítio S11 (floresta de várzea) para Dissomphalus e S8 (vegetação de taboca em floresta aberta de palmeiras nos terraços altos) para Pseudisobrachium (Tab, 2). Holepyris e Goniozus foram menos abundantes e diversificados, com apenas 2 e 1 morfo-espécies, respectivamente (Tab. 2). Anisepyris, Apenesia, Epyris, Rhabdepyris apresentaram abundância e diversidade intermediárias, com 13, 11,8 e 8 morfo-espécies respectivamente (Tab. 2).

De maneira geral, os Bethylidae foram um pouco mais abundantes $(58,8 \%)$ e apresentaram maior diversidade na região norte do PNSD, com 88 das 102 morfo-espécies $(86,3 \%)$, como todos os gêneros ocorrendo em ambas regiões, exceto Holepyris e Goniozus que foram coletados somente na região norte do Parque. Epyris foi o único gênero que ocorreu em mais sítios amostrais da região sul do que na norte (Tab. 2). A área sul do PNSD apresentou 41,2\% do total coletado, com 53 das 102 morfo-espécies do PNSD (52\%). Isto significa que, 39 morfo-espécies $(38,2) \%$ ocorreram em ambas regiões.

O sítio amostral S6 (floresta aberta de palmeiras nos terraços altos) apresentou maior abundância e diversidade de Bethylidae, seguido pelos sítios N9 (floresta de várzea), N6 (floresta aberta de palmeiras em depósitos coluviais) e N5 (floresta densa nos platôs), tanto em abundância como em diversidade (Tab. 2). Estes dois últimos sítios foram os que apresentaram o maior número de morfo-espécies exclusivas, 13,8 e 8 , respectivamente, sendo que o sítio S6 apresentou apenas 3 morfoespécies exclusivas (Tab. 2). Estes dados são muito semelhantes aos obtidos (ainda não publicados) para outras famílias de Hymenoptera como Chalcididae, Eucharitidae, Evaniidae, Mutillidae, Pompilidae, Sphecidae e Vespidae, cuja ordem decrescente de riqueza foi N6, S6, N9 e N5, apenas Meliponinae diferiu um pouco, cuja ordem decrescente de riqueza foi N6, N8, N5 e N1.

Os sítios que apresentaram maior importância, baseada em $\left(I_{i}+Q_{m i}\right) / 2$, quando consideradas as morfoespécies e gêneros foram predominantemente aqueles localizados na região norte, como N3, N5, N6 e N9 (Tab. 5). Locais com baixo número de táxons que foram compartilhados com outros locais, como o sítio S8 (Vegetação de taboca em floresta aberta de palmeiras nos terraços altos) tiveram valor de importância baixo para a análise tanto de morfo-espécies como de gêneros.

Das 102 morfo-espécies encontradas, 49 delas ocorreram em um único sítio, representado $48 \%$ do total. Estas morfo-espécies 
Tabela 2. Número de exemplares de morfo-espécies de Bethylidae nos sítios amostrais do Parque Nacional da Serra do Divisor, Acre. Ps. $=$ Pseudisobrachium, Ap. $=$ Apenesia, Di. $=$ Dissomphalus, Ep. $=$ Epyris, Rh. $=$ Rhabdepyris, An. $=$ Anisepyris, Ho. $=$ Holepyris, Go. $=$ Goniozus, $\mathrm{N}=$ Norte, $\mathrm{S}=\mathrm{Sul}$; Riq. $\mathrm{S}=$ riqueza de morfo-espécies.

\begin{tabular}{|c|c|c|c|c|c|c|c|c|c|c|c|c|}
\hline & & & & & Sític & ams & rais & & & & & \\
\hline Táxon & N1 & N3 & N5 & N6 & N8 & N9 & S1 & S4 & S6 & S8 & $\mathrm{S} 10$ & $\mathrm{~S} 11$ \\
\hline Ps. 1 & & & & & & 2 & & 1 & 5 & & 1 & \\
\hline Ps. 2 & & & 4 & & & & & 1 & 2 & & 1 & \\
\hline Ps. 3 & & & 1 & & & 2 & & & & & & \\
\hline Ps 4 & & & & & & & 1 & & & & & \\
\hline Ps. 5 & & & & & 1 & & & 3 & 3 & & & \\
\hline Ps. 6 & & & & 1 & & & & & 1 & & 1 & 1 \\
\hline Ps, 7 & & & & & & & 2 & & 1 & & & \\
\hline Ps. 8 & & 1 & & 3 & & & 2 & & 4 & & 2 & \\
\hline Ps. 9 & 1 & & 3 & 1 & & & 2 & & 2 & & & \\
\hline Ps, 10 & & & & 2 & & 1 & 1 & & 2 & & & \\
\hline Ps. 11 & & & & 2 & & & & & & & & \\
\hline Ps. 12 & & & & & & 1 & & & & & & \\
\hline Ps. 13 & & & & & & & 1 & 1 & 1 & & & 2 \\
\hline Ps. 14 & & & & 5 & & & & & & & & \\
\hline Ps. 15 & & & & 1 & & & & & & & 2 & \\
\hline Ps. 16 & 2 & 1 & & 4 & & & & & 9 & & 1 & \\
\hline Ps. 17 & & 1 & & 3 & & & & & & & & \\
\hline Ps. 18 & & & & 1 & & & & 1 & 1 & & & \\
\hline Ps. 19 & & & & 8 & 1 & 1 & & 1 & 1 & & & \\
\hline Ps. 20 & & & & 3 & & 6 & 2 & & 1 & & & \\
\hline Ps. 21 & & & & 1 & & & & & 1 & & & \\
\hline Ps. 22 & & & & 1 & & & & & & & & \\
\hline Ps. 23 & & 1 & & & & & & & & & & \\
\hline Ps. 24 & & & & 2 & & & & & & & & \\
\hline Ps. 25 & & & 1 & & 1 & & & & 1 & & & \\
\hline Ps. 26 & 1 & 1 & 1 & 1 & & & & & & & 1 & \\
\hline Ps. 27 & & & 1 & & & & & & 1 & & & \\
\hline Ps. 28 & & 1 & & & & & & & & & & \\
\hline Ps. 29 & 1 & & & & & & & & & & & \\
\hline Ap. 1 & & & & & & 1 & & & & & 1 & \\
\hline Ap. 2 & & & & 1 & 2 & 1 & & & & & & \\
\hline Ap. 3 & & & 1 & & & 4 & & & & & & \\
\hline Ap. 4 & 1 & & 1 & & & & & & 2 & & & \\
\hline Ap. 5 & 1 & & & & & & & & & & & \\
\hline Ap. 6 & & 3 & & & & & & 1 & & & & \\
\hline Ap. 7 & & & & 1 & & & & & & & & \\
\hline Ap. 8 & & & 1 & & & & & & 1 & & 1 & \\
\hline Ap. 9 & & 1 & & & & & 1 & & 1 & & & \\
\hline Ap. 10 & & 1 & & & & & & & 1 & & & \\
\hline
\end{tabular}


Tabela 2. Continuação

\begin{tabular}{|c|c|c|c|c|c|c|c|c|c|c|c|c|}
\hline \multicolumn{13}{|c|}{ Sitios amostrais } \\
\hline Táxon & N1 & N3 & N5 & N6 & N8 & N9 & S1 & S4 & S6 & 58 & S10 & $\mathrm{S} 11$ \\
\hline Ap. 11 & & & & 1 & & & & & & & & \\
\hline Di. 1 & & & & & & 1 & & & 1 & & & \\
\hline Di. 2 & & & & & & & & & 1 & & 2 & \\
\hline Di. 3 & & & & & & & & 1 & & & & \\
\hline Di. 4 & & 1 & & & & 5 & 1 & & 2 & & & \\
\hline Di. 5 & 1 & & & & & & 1 & & 4 & & 1 & \\
\hline Di. 6 & 1 & & & & & & & & & & & \\
\hline Di. 7 & & & & & & 1 & & & & & & \\
\hline Di. 8 & & 1 & & & & & & & & & & \\
\hline Di. 9 & & & & & & & & & & & 1 & \\
\hline Di. 10 & & & 2 & & & & & & & & & \\
\hline Di. 11 & & & 1 & & & & & & & & & \\
\hline Di. 12 & & & 1 & & & & 1 & & 19 & & 1 & \\
\hline Di. 13 & & & 1 & & 2 & & 2 & 2 & & & & \\
\hline Di. 14 & & & 1 & & & & & & & & & \\
\hline Di. 15 & & & 2 & & & & & & & & & \\
\hline DI. 16 & & & & 2 & 2 & & & 3 & 1 & 1 & 1 & \\
\hline Di. 17 & & & 3 & 1 & & & & & & & & \\
\hline Di. 18 & & & & 1 & & 1 & & & 4 & & 2 & \\
\hline DI. 19 & & & & & & 2 & & & & & & \\
\hline Di. 20 & & & & & & & & & & & 1 & \\
\hline Di. 21 & & & & & & 3 & & & & & & \\
\hline Di. 22 & & 1 & & & & & & 1 & & & & \\
\hline Di. 23 & & & 1 & & & & & & & & 1 & \\
\hline Di. 24 & & & 2 & 1 & & 1 & & 1 & 1 & & & \\
\hline Di. 25 & & & & & 1 & & & & & & & \\
\hline Di. 26 & & & & & 1 & 1 & & 1 & 1 & & 1 & \\
\hline Di. 27 & & & & & & 1 & & & & & & \\
\hline Di. 28 & & & & 1 & 1 & 1 & & & & & & \\
\hline Di. 29 & & 1 & & & & & & & 1 & & & \\
\hline Di. 30 & & & & & 1 & & & & 2 & & & \\
\hline Ep. 1 & & & & & & & & & & & 1 & 1 \\
\hline Ep. 2 & & & & & & & & & 1 & & & \\
\hline Ep. 3 & & & & 1 & & 6 & & & & & & \\
\hline Ep. 4 & & & 1 & & & 1 & & & 1 & & & \\
\hline Ep. 5 & & & 1 & & & & & & & & & \\
\hline Ep. 6 & & & & & & 1 & & & & & & \\
\hline Ep. 7 & & & & & & 2 & & & & & & \\
\hline Ep. 8 & & & & & & & & & 1 & & 1 & \\
\hline Ep. 9 & & & 1 & & & & & & & & & \\
\hline Ep. 10 & & & & & & 1 & & & & & & \\
\hline Ep. 11 & & & & 1 & & & & & & & & \\
\hline Ep. 12 & & & & & & & & & & & 1 & \\
\hline Ep. 13 & & & & & & & 1 & & & & & \\
\hline Rh. 1 & & 1 & & & & & & & & & & \\
\hline Rh. 2 & & & 1 & & & & & & & & & \\
\hline Rh. 3 & & & 1 & & & 2 & & & 1 & & & \\
\hline Rh. 4 & & & & 1 & & & & & 2 & & 1 & \\
\hline Rh. 5 & & & & & & 1 & & & & & & \\
\hline Rh. 6 & & & 1 & 1 & & & & & & & & \\
\hline Rh. 7 & & & & 2 & & 1 & & & 3 & & & \\
\hline Rh. 8 & & & & & & & & & 3 & & & \\
\hline An. 1 & & & & & & 1 & & & & & & \\
\hline An. 2 & & & & & & 1 & & & & & & \\
\hline An. 3 & & 1 & & & & 1 & & & & & & \\
\hline An. 4 & & & & & & & & & 1 & & & \\
\hline An. 5 & & 1 & & & & & & & & & & \\
\hline An. 6 & & & & & & 6 & & & & & & \\
\hline An. 7 & & & & 2 & & & & & & & & \\
\hline An. 8 & & & & & & 4 & & & & & & \\
\hline Ho. 1 & & 1 & & & & & & & & & & \\
\hline Ho. 2 & & & & & 1 & & & & & & & \\
\hline Go. 1 & & & 1 & & & & & & & & & \\
\hline Riq. $S$ & 8 & 17 & 25 & 30 & 11 & 32 & 13 & 13 & 39 & 1 & 22 & 3 \\
\hline
\end{tabular}


apresentaram valores de $\mathrm{Q}_{j}$ superior a 0,9 (Tab, 3). As morfo-espécies com $\mathrm{Qj}=0,5$ correspondem a $3 \%$ dos casos e são as que apresentam menor prioridade de conservação (Tab. 3). Isto ocorreu por que índice de prioridade de conservação dos táxons é inversamente proporcional ao número de sítios amostrais em que eles são coletados, então quanto maior for o número de sítios amostrais em que um táxon é coletado, menor será sua prioridade de conservação. Este dado é esperado, uma vez que táxons com maior distribuição em uma área possuem maior capacidade de sobrevivência em casos de impactos.

Considerando-se as morfo- espécies agrupadas em nível de gênero (Tab. 4), aquele com maior prioridade de conservação é o gênero Goniozus. Quando se considera os sítios das regiões sul e norte do PNSD separados, Goniozus apresenta maior prioridade na região norte e Anisepyris na região sul (Tab. 4), já que Holepyris e Goniozus não foram detectados na região norte (Tab. 2). O único gênero que não apresenta prioridade de conservação é Pseudisobrachium pois ocorreu em todos os sítios. Quando se considera a região norte isoladamente, Pseudisobrachium, Apenesia e Dissomphalus apresentaram prioridade de conservação igual a zero. Já na região sul, apenas Pseudisobrachium

Tabela 3. Valores de Prioridade de Conservação(Qj) para os 102 morfo-espécies de Bethylidae do Parque Nacional da Serra do Divisor.

\begin{tabular}{ccccccccccc}
\hline Dezenas & \multicolumn{10}{c}{ Unidades das morfo-espécies } \\
\hline & 0 & 1 & 2 & 3 & 4 & 5 & 6 & 7 & 8 & 9 \\
\hline 1 & 0,667 & 0,917 & 0,917 & 0,667 & 0,917 & 0,833 & 0,667 & 0,833 & 0,750 & 0,583 \\
2 & 0,601 & 0,833 & 0,917 & 0,917 & 0,917 & 0,750 & 0,667 & 0,833 & 0,917 & 0.917 \\
3 & 0,833 & 0,750 & 0,750 & 0,833 & 1,000 & 0,833 & 0,917 & 0,750 & 0,750 & 0,833 \\
4 & 0,917 & 0,833 & 0,833 & 0,917 & 0,667 & 0,750 & 0,917 & 0,917 & 0,917 & 0,917 \\
5 & 0,917 & 0,917 & 0,667 & 0,667 & 0,917 & 0,917 & 0,500 & 0,833 & 0,667 & 0,917 \\
6 & 0,917 & 0,917 & 0,833 & 0,833 & 0,583 & 0,917 & 0,583 & 0,917 & 0,750 & 0,833 \\
7 & 0,833 & 0,833 & 0,917 & 0,833 & 0,750 & 0,917 & 0,917 & 0,917 & 0,833 & 0,917 \\
8 & 0,917 & 0,917 & 0,917 & 0,917 & 0,917 & 0,917 & 0,750 & 0,750 & 0,917 & 0,833 \\
9 & 0,750 & 0,917 & 0,917 & 0,833 & 0,917 & 0,917 & 0,917 & 0,917 & 0,917 & 0,917 \\
10 & 0,917 & 0,917 & 0,917 & & & & & & & \\
\hline
\end{tabular}


teve prioridade zero. Isto ocorreu por que táxons menos abundantes e mais diversificados influenciaram positivamente o índice de prioridade de conservação de táxons.

Em nível de gênero, a ordem de prioridade de conservação encontrada para o PNSD foi Goniozus $(0,917)$, Holepyris $(0,833)$, Anisepyris $(0,667)$, Rhabdepyris $(0,500)$, Epyris $(0,417)$, Apenesia $(0,167)$, Dissomphalus $(0,083)$ e Pseudisobrachium $(0,000)$ (Tab. 4).

Com os dados acima apresentados, foi possível estabelecer uma ordem de importância dos sítios para conservação (Tab. 6), em que os dois sítios de maior importância relativa para Bethylidae têm como cobertura florestas densas (N5 e N3), que tem vegetação com fisionomia mais complexas. A maior coerência entre os três métodos $I_{i}, Q_{m i}$ e $\left(I_{i}+\right.$ $\left.Q_{m i}\right) / 2$ foi observada quando se calcula a importância dos sítios em separado e por gênero e a menor quando se calcula utilizando as morfo-espécies.
Isto ocorre justamente por que análises com dados de espécie apresentam muitos números baixos para cada táxon, o que normalmente não acontece com análises em nível de gêneros. O uso de gêneros (Azevedo \& Helmer, 1999) ou mesmo família (Marinoni \& Dutra, 1997) tem demonstrado ser uma ferramenta boa para análises de estrutura de comunidade de insetos.

Nossas análises reforçam a possibilidade do uso de levantamento ecológico rápido para estimar riqueza de espécies e corroboram com metodologia proposta por Oliver \& Beattie (1996) que utilizam morfoespécie para inventariar a biodiversidade.

\section{CONCLUSÕES}

Com base no presente estudo, concluímos que foi possível discriminar sítios com graus diferentes de diversidade usando-se os

Tabela 4. Cálculo de $\mathrm{Q}_{\mathrm{j}}$ utilizando dados de gênero.

\begin{tabular}{lccc}
\hline \multicolumn{1}{c}{ Gênero } & Todo PNSD & Norte & Sul \\
\hline Pseudisobrachium & 0,000 & 0,000 & 0,000 \\
Apenesia & 0,167 & 0,000 & 0,333 \\
Dissomphalus & 0,083 & 0,000 & 0,167 \\
Epyris & 0,417 & 0,500 & 0,333 \\
Rhabepyris & 0,500 & 0,333 & 0,667 \\
Anisepyris & 0,667 & 0,500 & 0,833 \\
Holepyris & 0,833 & 0,667 & 1 \\
Goniozus & 0,917 & 0,833 & 1 \\
\hline
\end{tabular}

' -Estes gêneros não ocorreram na regiâo Sul. 
Bethylidae; os sítios com maior importância relativa são as da região norte do PNSD, em particular os com florestas densas; os táxons com maior prioridade de conservação são aqueles que ocorrem em apenas um sítio amostral, e que a análise em nível de gênero foi mais consistente do que em morfo-espécie.

\section{AGRADECIMENTOS}

À Fundação S.O.S. Amazônia, The Nature Conservancy e IBAMA pela oportunidade de participação no Projeto Para a Conservação da Serra do Divisor. À Dra. Verônica Telma da Rocha Passos (Universidade Federal do Acre/Instituto de Meio Ambiente

Tabela 5. Valores da importância dos sítios utilizando morfo-espécie e gêneros para a área total e apenas gêneros para as regiões norte e sul.

\begin{tabular}{|c|c|c|c|c|c|c|c|c|c|}
\hline \multirow[t]{2}{*}{ Sítio } & \multicolumn{3}{|c|}{ Morfo-espéciesÁrea Total } & \multicolumn{3}{|c|}{ GêneroÁrea Total } & \multicolumn{3}{|c|}{$\begin{array}{c}\text { GênerosNorte e Sul } \\
\text { Separados }\end{array}$} \\
\hline & 1 & $Q_{m} i$ & $\left(1+Q_{m i}\right) / 2$ & I & $Q_{\text {mii }}$ & $\left(I_{i}+Q_{m i}\right) / 2$ & $I_{1}$ & $Q_{r i t}$ & $\left(I_{1}+Q_{m i v}\right) / 2$ \\
\hline $\mathrm{N} 1$ & 0,087 & 0,815 & 0,451 & 0,070 & 0,083 & 0,076 & 0,000 & 0,000 & 0,000 \\
\hline N3 & 0,163 & 0,814 & 0,4885 & 0,628 & 0,375 & 0,502 & 0,529 & 0,250 & 0,390 \\
\hline N5 & 0,236 & 0,800 & 0,518 & 0,581 & 0,347 & 0,464 & 0,588 & 0,278 & 0,433 \\
\hline N6 & 0,271 & 0,767 & 0,519 & 0,512 & 0,306 & 0,409 & 0,471 & 0,222 & 0,346 \\
\hline N8 & 0,094 & 0,727 & 0,410 & 0,302 & 0,271 & 0,286 & 0,235 & 0,167 & 0,201 \\
\hline N9 & 0,303 & 0,802 & 0,552 & 0,512 & 0,306 & 0,409 & 0,471 & 0,222 & 0,346 \\
\hline S1 & 0,111 & 0,724 & 0,417 & 0,186 & 0,167 & 0,176 & 0,357 & 0,208 & 0,282 \\
\hline S4 & 0,106 & 0,692 & 0,399 & 0,070 & 0,083 & 0,076 & 0,214 & 0,167 & 0,190 \\
\hline S6 & 0,348 & 0,738 & 0,543 & 0,512 & 0,306 & 0,409 & 1,000 & 0,389 & 0,694 \\
\hline S8 & 0,006 & 0,500 & 0,253 & 0,023 & 0,042 & 0,032 & 0,071 & 0,083 & 0,077 \\
\hline S10 & 0,193 & 0,742 & 0,4675 & 0,326 & 0,233 & 0,280 & 0,643 & 0,300 & 0,472 \\
\hline S11 & 0,026 & 0,722 & 0,374 & 0.116 & 0,208 & 0,162 & 0,143 & 0,167 & 0,155 \\
\hline
\end{tabular}

Tabela 6. Ordem de importância dos sítios para conservação, em função da forma de agrupamentos de dados utilizada.

\begin{tabular}{|c|c|c|c|c|c|c|c|c|c|}
\hline \multirow[t]{2}{*}{ Ordem } & \multicolumn{3}{|c|}{ Morfo-espéciesÁrea Total } & \multicolumn{3}{|c|}{ GêneroÁrea Total } & \multicolumn{3}{|c|}{$\begin{array}{c}\text { GênerosNorte e Sul } \\
\text { Separados }\end{array}$} \\
\hline & I & $Q_{m} i$ & $\left(I_{1}+Q_{m u}\right) / 2$ & I & $Q_{m !}$ & $\left(I_{1}+Q_{m i}\right) / 2$ & $I_{1}$ & $Q_{\mathrm{rin}}$ & $\left(1_{1}+Q_{m i t}\right) / 2$ \\
\hline 1 & S6 & N1 & N9 & N3 & N3 & N3 & N5 & N5 & N5 \\
\hline 2 & N9 & N3 & S6 & N5 & N5 & N5 & N3 & N3 & N3 \\
\hline 3 & N6 & N9 & N6 & N6 & N6 & N6 & N6 & N6 & N6 \\
\hline 4 & N5 & N5 & N5 & N9 & N9 & N9 & N9 & N9 & N9 \\
\hline 5 & $\mathrm{~S} 10$ & N6 & N3 & S6 & 56 & S6 & N8 & N8 & N8 \\
\hline 6 & N3 & $\mathrm{S} 10$ & S10 & S10 & N8 & N8 & N1 & $\mathrm{N} 1$ & N1 \\
\hline 7 & S1 & S6 & $\mathrm{N} 1$ & N8 & S10 & S10 & S6 & S6 & $\mathrm{S} 6$ \\
\hline 8 & $\$ 4$ & N8 & S1 & S1 & S11 & S1 & S10 & S10 & $\mathrm{S} 10$ \\
\hline 9 & N8 & S1 & N8 & S11 & S1 & S11 & S1 & S1 & $\mathrm{S} 1$ \\
\hline 10 & N1 & $\mathrm{S} 11$ & $\mathrm{~S} 4$ & $\mathrm{~N} 1$ & N1 & $\mathrm{N} 1$ & S4 & S4 & S4 \\
\hline 11 & $\mathrm{~S} 11$ & S4 & S11 & S4 & $\mathrm{S} 4$ & S4 & $\mathrm{S}_{11}$ & S11 & S11 \\
\hline 12 & S8 & $\mathrm{S} 8$ & S8 & S8 & S8 & S8 & S8 & S8 & S8 \\
\hline
\end{tabular}


do Acre) pelo auxílio na elaboração dos dados descritivos da área de estudo e apoio logístico.

\section{Bibliografia citada}

Azevedo, C.O. 1999. Bethylidae, In: Brandão, C.R.F.; E.M. Cancello (Eds.). Biodiversidade do estado de São Paulo, Brasil: síntese do conhecimento ao final do século XX. Vol. 5: Invertebrados Terrestres. São Paulo, FAPESP, p.169-181.

Azevedo, C.O.; Helmer, J.L. 1999. Ecologia de comunidade de Bethylidae (Hymenoptera, Chrysidoidea) da Reserva Biológica do Roncador, Brasília, DF, Brasil. Revista Brasileira de Zoologia 16(4):1115-1126.

Brasil, 1977. Projeto RADAMBRASIL: levantamento de recursos naturais.V.13. Folhas SB/SC. 18. Javari/Contamana. Rio de Janeiro.

Evans, H. E. 1964. A synopsis of the American Bethylidae (Hymenoptera, Aculeata). Bulletin of the Museum of Comparative Zoology 132(1):1-222.

Goldstein, P.Z. 1997. How many things are there? A reply to Oliver and Beattie, Beattie and Oliver, Oliver and Beattie, and Oliver and Beattie. Conservation Biology 1I(2):571-574.

LaSalle, J.; Gauld, I. D. 1991. Parasitic Hymenoptera and the biodiversity crisis. Redia 74(3):315-334.

Marinoni, R.C.; Dutra, R.R.C. 1997. Famílias de Coleoptera capturadas com armadilha Malaise em oito localidades do estado do Paraná, Brasil. Diversidade alfa e beta. Revista Brasileira de Zoologia 14(3):751-770.

Minns, C.K. 1987. A methods of ranking species and sites for conservation using presence-absence data and its application to native freshwater fish in New Zealand. New Zealand Journal of Zoology 14:43-49.
New, T.R. 1999. Entomology and nature conservation. European Journal of Entomology 96:11-17.

Noyes, J.S. 1989. A study of five methods of sampling Hymenoptera (Insecta) in a tropical rainforest, with special reference to the Parasitica. Journal of Natural History 23:285-298.

Oliver, I.; Beattie, A.J. 1993. A possible method for the rapid assessment of biodiversity. Conservation Biology 7(3):562-568.

Oliver, I.; Beattie, A.J. 1996. Invertebrate morphospecies as surrogates for species: a case study. Conservation Biology 10(1):99-109.

Rathcke, B.J.; Price, P.W. 1976. Anomalous diversity of tropical ichneumonid parasitoids: a predator hypothesis. The American Naturalist 110:889-893. 